\title{
Application-Centric, Energy-Efficient Network Architecture ACTION, Based on Virtual Optical Slice Core and Deterministic Optical Access Network
}

\author{
Kunitaka Ashizawa $^{1}$, Satoru Okamoto ${ }^{2,1}$, Naoaki Yamanaka ${ }^{1}$, Eiji Oki ${ }^{2}$, Andrea Fumagalli ${ }^{3}$, \\ and Malathi Veeraraghavan ${ }^{4}$ \\ ${ }^{1}$ Department of Information and Computer Science, Keio University, 3-14-1 Hiyoshi, Kohoku, \\ Yokohama, Kanagawa 223-8522, Japan \\ ${ }^{2}$ The University of Electro-Communications, 1-5-1 Chofugaoka, Chofu, Tokyo 182-8585, Japan \\ ${ }^{3}$ The University of Texas at Dallas, 800 W. Campbell Road, Richardson, Texas 75080-3021, USA \\ ${ }^{4}$ University of Virginia, 351 McCormick Rd VA 22904-4743, USA
}

(Received February 24, 2015 : revised May 26, 2015 : accepted May 29, 2015)

\begin{abstract}
The Internet traffic is forecasted to grow at a compound annual rate of $21 \%$ from 2013 to 2018, according to surveys carried out by Cisco [1]. Network resources are significantly over-provisioned in today's networks, and it is quite common to see link utilization in the $30-40 \%$ range [2]. Additionally, the multi-media services have widely divergent bandwidth and Quality of Experience (QoE) requirements. Unfortunately, the huge transmission capacity will increase the power consumption of network equipment [3]. Applications Coordinated with Transport, Internet Protocol and Optical Networks (ACTION) [4] has been proposed to realize a multi-QoE, application-centric, and highly energy-efficient network that leverages flexible elastic optical network technologies [5-7]. This paper provides key network technologies for realizing the ACTION, which are a virtual optical slice core network and a Time Division Multiplexing (TDM)-based deterministic active optical access network.
\end{abstract}

Keywords: Quality of experience, Quality of service, Quality of transport, Flexible elastic network OCIS codes : (060.0060) Fiber optics and optical communications; (060.4510) Optical communications

\section{INTRODUCTION}

Today's Internet supports a varied set of network services, including private line, mobile, residential Internet access, and inter-datacenter network, by simply over-provisioning links, e.g., $30 \%-40 \%$ link utilization levels are quite common [2]. However low network utilization results in low energy efficiency levels, and thus increasing energy efficiency is an important requirement for future network designs. Therefore, in order to realize a multi-QoE, application-centric, and highly energy-efficient network, Applications Coordinated with Transport, Internet Protocol and Optical Networks (ACTION) [4], which leverages flexible elastic optical network technologies [5-7], has been proposed.

In this paper, we introduce a virtual optical slice core network and a Time Division Multiplexing (TDM)-based deterministic active optical access network in order to realize ACTION. The virtual optical slice core network can guarantee multi Quality of Services (QoS) by adjusting elastic optical pipe bandwidth and can provide an energy efficient network by adopting the Multi-(layer, path, and resources) Dynamically Optimized Routing (MiDORi) technique [8-13], which aggregates traffic onto a small number of links and powers off the routers that are not used to convey traffic. Also, the edge nodes of the core network, called ACTION edges, monitor application Quality of Experience (QoE) and assign flows to various multi-QoS slices. The TDM-based deterministic active optical access network can guarantee the various QoE requirements of each user in the access network based on QoS levels in the core network. It adopts the Optical Distribution network (ODN) by adopting Mach-Zehnder (MZ) type Plumbum Lanthanum Zirconate Titanate (PLZT) optical switches [14-16], which can support unicast and multicast states, in order to provide multi services and energy efficient network services, according to the user's requirements.

\footnotetext{
*Corresponding author: Kunitaka.Ashizawa@yamanaka.ics.keio.ac.jp

Color versions of one or more of the figures in this paper are available online.
} 


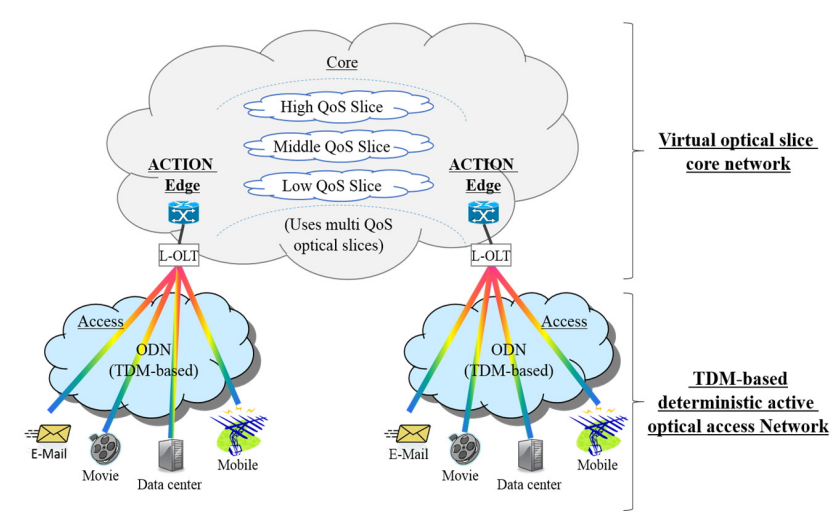

FIG. 1. ACTION architecture overview.

\section{ACTION ARCHITECTURE}

There are mainly three problems in the today's Internet: "users requiring different QoE levels", "application divergence", and "overprovisioning (which results in higher energy consumption levels)".

Figure 1 shows the ACTION architecture [4], including the virtual optical slice core network and the TDM-based deterministic optical access network, which are introduced in this paper, in order to solve the above problems. This core network uses multi QoS optical slices to create multiple bandwidth pipes between nodes [5-7]. The ACTION edge nodes of the core network monitor each application's QoE. This access network is comprised of the Logical-Optical Line Terminals (L-OLTs) [17], which provide functions suited for each network service, and the ODN, which adopts the MZ type PLZT optical switches [14-16].

\section{VIRTUAL OPTICAL SLICE CORE NETWORK}

Figure 2 shows the virtual optical slice core network, where multi-route paths can be aggregated into several virtual optical paths, including high, middle, and low QoS optical slices. The multi-QoS optical slices are established by the self-sizing elastic network technique [18]. All the virtual link utilization rates are monitored, and their bandwidth levels are adjusted to hit the utilization target. By using this technique, the link bandwidth can be adjusted to traffic levels automatically, and if needed, packets can be transmitted on parallel routes.

In order to reduce power consumption in the physical network, this core network introduces the MiDORi [8-13] technique. The MiDORi aggregates multiple traffic flows onto a small number of physical routes, creating highly utilized links wherever possible, and instructs the router to power-off unused interfaces whenever possible, as shown in Fig. 3.

Additionally, the MiDORi technique adopts the practical calculation algorithm determining an energy optimal topology

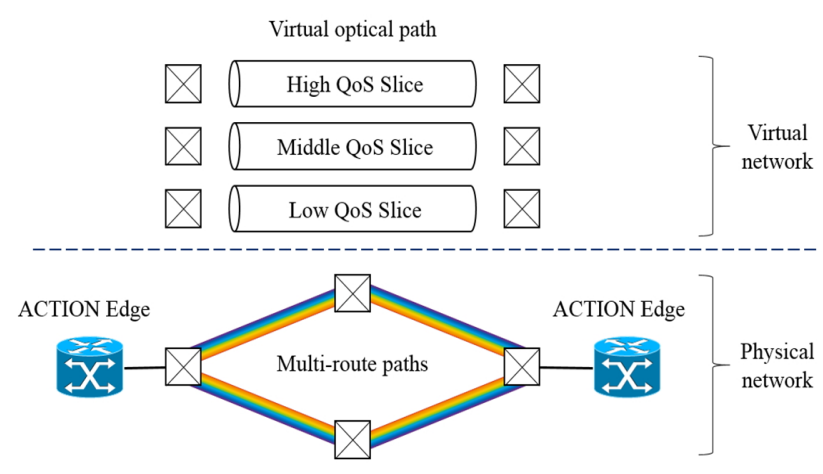

FIG. 2. Virtual optical slice core network.
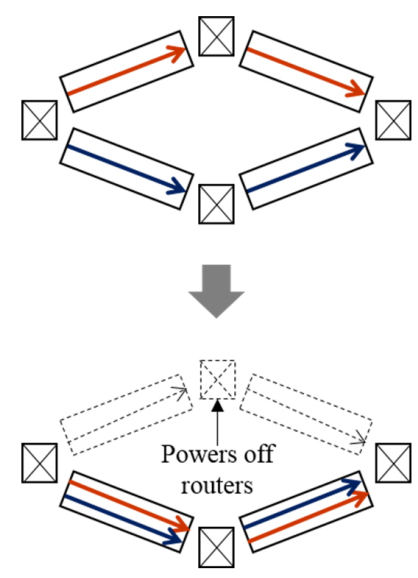

FIG. 3. MiDORi technique.

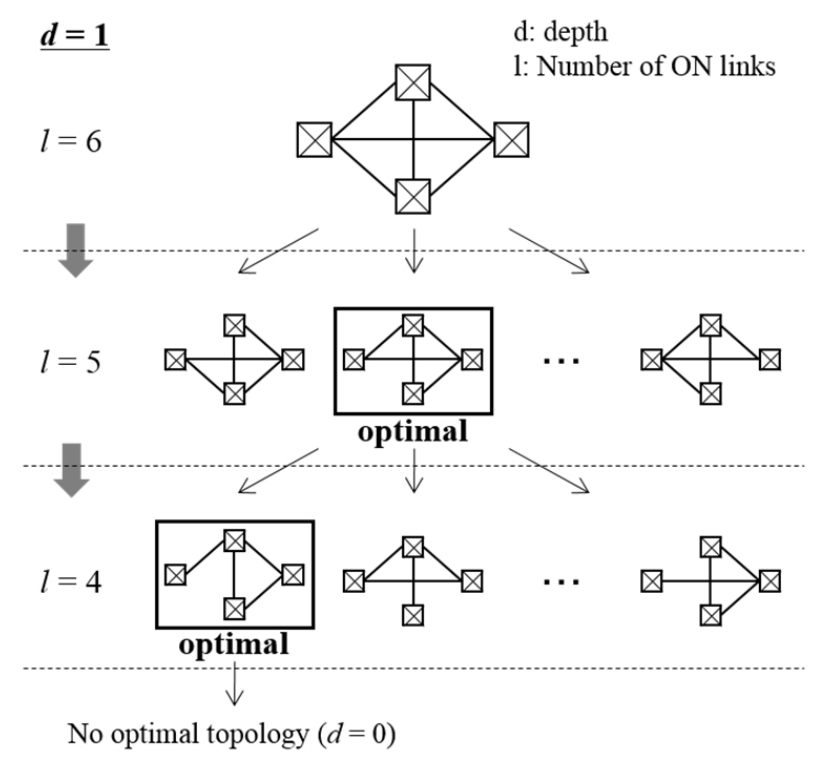

FIG. 4. Practical calculation algorithm determining an energy optimal topology.

[10], as represented in Fig. 4. This algorithm iterates the steps of replacing the topology with a local optimum topology with $d$ links powered off. The detailed operation 


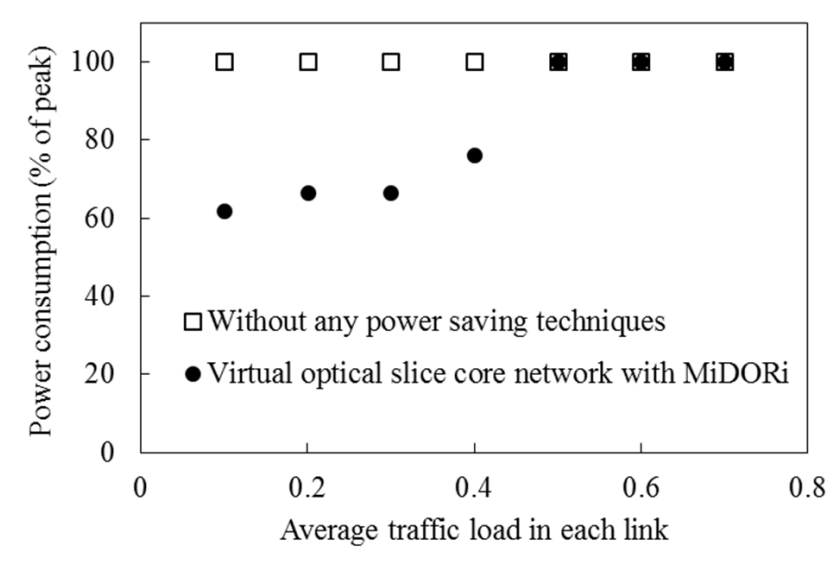

FIG. 5. Power consumption of peak versus average traffic load in each link.

of this algorithm is described below from Step 1 to Step 7.

1. Set the depth $(d)$ of this algorithm. $d$ is an integer value that changes from 1 to $n-1$ ( $n$ is the total number of nodes).

2. Generate several patterns of topologies powering off $d$ links from the original topology.

3. Check if each generated topology satisfies the QoS requirement and exclude those that do not. If no topologies meet the requirement, decrement $d$ and return to Step 2.

4. Calculate the maximum link utilization for each remaining topology.

5. Select one topology that has the minimum value $(m)$ of maximum link utilization and replace it with the original topology. If $m$ exceeds 1.0 , decrement $d$ and return to Step 2.

6. Iterate from Step 2 to Step 5, while the connectivity of nodes is maintained. If not, decrement $d$ and iterate the steps.

7. Stop all steps when $d$ is decremented to 0. The remaining topology is decided as the energy optimal topology to be reconfigured.

Performance Evaluation for power consumption in the virtual optical slice core network: The simulation parameters are shown below. The topology assumed is the NSFnet, which has 14 nodes and 20 links. The link capacity is 1.0 for all links. The maximum traffic load for each link is 0.9. The depth $(d)$ in the algorithm of the MiDORi is set to 1. Figure 5 shows the power consumption of peak versus average traffic load in each link. The virtual optical slice core network with the MiDORi reduces the power consumption by up to 38 $\%$, compared to without any power saving techniques.

\section{ACTION EDGE}

Figure 6 shows the basic function of an ACTION Edge

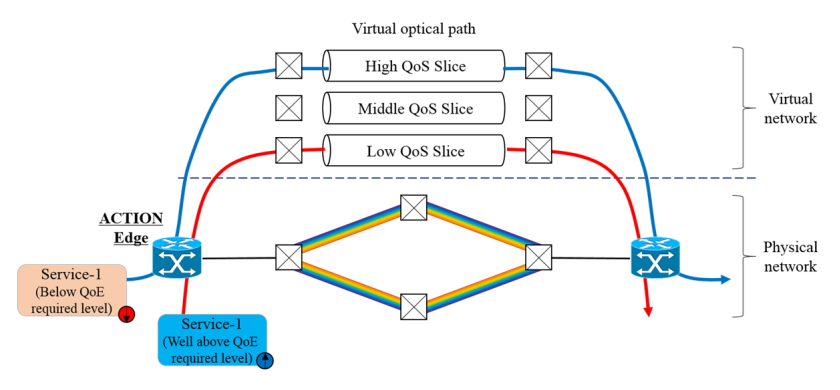

FIG. 6. Function of ACTION Edge.

of the core network. It monitors the QoE of certain applications and modifies the transport network bandwidth automatically. If the QoE degradation is detected, the application's flow is mapped to a slice with better QoS. On the other hand, if QoE is well above the required level, a lower QoS slice may be used.

However, the ACTION Edge has mainly two problems to be solved, including monitoring all TCP flows between source-destination pairs, especially in high-speed networks, and determining the desired $\mathrm{QoE}$ for each application.

In order to solve the first problem, we target $\alpha$-flows, which are used for large-sized, high-rate flows [19, 20], for identification and for the provision of QoE assurance. Hybrid Network Traffic Engineering System (HNTES) technique [19], which is an intra-domain solution, can be used. This technique performs two tasks: (i) enables the automatic identification of flows at a provider network's ingress routers, where source and destination address prefixes of observed $\alpha$-flows can be used to configure firewall filter rules for future $\alpha$-flow redirection, and (ii) redirects these flows to trafficengineered QoS-controlled virtual circuits by using a no-policing and queuing solution with Weighted Fair Queueing (WFQ) and Priority Queueing (PQ), which are both sufficient and the best for this application.

In order to solve the second problem, we propose two methods: (i) an explicit method using signaling, and (ii) an implicit method by having a proxy function set QoE values at the ACTION Edge. The sender application can keep increasing the flow rate until the required QoE is met.

\section{TDM-BASED DETERMINISTIC ACTIVE OPTICAL ACCESS NETWORK}

Figure 7 shows the TDM-based deterministic active optical access network used to guarantee QoE requirements of each user based on the QoS levels available in the core network. This network is comprised of the L-OLTs [17], the LogicalOptical Network Units (L-ONUs), and the configurable ODN. Both L-OLT and L-ONU are structured by using reconfigurable logical devices and provide media access control (MAC) functions and physical (PHY) functions suited for each network service. In order to provide multi services and energy efficient network services, the ODN 
introduces the MZ type PLZT optical switches [14-16], which can switch an optical signal at nano-second speed (5-10 ns) and support unicast and multicast states. The switch consists of $1 \times N$ PLZT optical switches, which in turn consist of $1 \times 2$ PLZT optical switches in a multistage configuration.

The MZ-type PLZT optical switch can yield the low-power splitter mode by applying no electric power to the switch, in addition to the normal switch modes which have bar and cross states of optical signal. Figure 8 shows the structure of a PLZT optical switch. In the normal switch modes, the optical signal is output in $\mathrm{X}$ port by applying the voltage

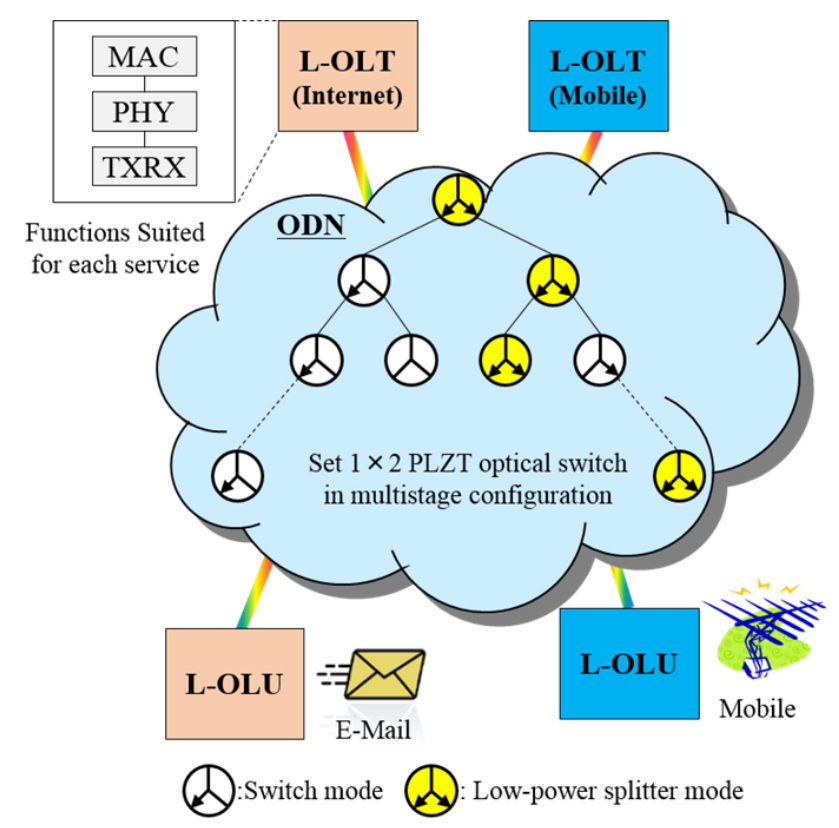

FIG. 7. TDM-based deterministic active optical access network.

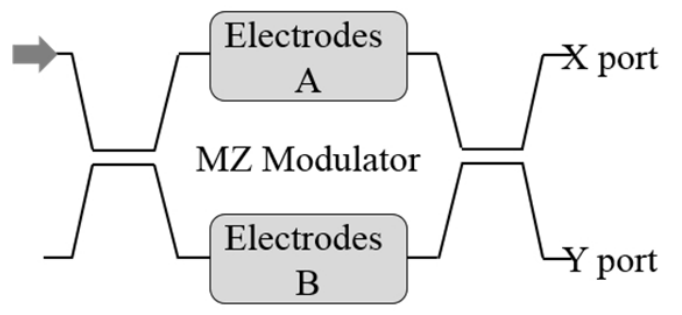

\begin{tabular}{|c|c|c|c|}
\hline \# & $\begin{array}{l}\text { Mode of PLZT } \\
\text { optical switch }\end{array}$ & $\begin{array}{l}\text { Voltage } \\
\text { difference }\end{array}$ & $\begin{array}{l}\text { Optical } \\
\text { insertion } \\
\text { gain }\end{array}$ \\
\hline & $\begin{array}{l}\text { Switch mode } \\
\text { (Bar state) }\end{array}$ & $\begin{array}{l}\mathrm{A}, 0.0 \mathrm{~V} \\
\mathrm{~B}, 8.0 \mathrm{~V}\end{array}$ & $\begin{array}{l}X, 0 d B \\
Y,-10.9 d B\end{array}$ \\
\hline (b) & $\begin{array}{l}\text { Low-power } \\
\text { splitter mode }\end{array}$ & $\begin{array}{l}\mathrm{A}, 0.0 \mathrm{~V} \\
\mathrm{~B}, 0.0 \mathrm{~V}\end{array}$ & $\begin{array}{l}X,-3 d B \\
Y,-3 d B\end{array}$ \\
\hline (c) & $\begin{array}{l}\text { Switch mode } \\
\text { (Cross state) }\end{array}$ & $\begin{array}{l}\text { A, } 10.5 \mathrm{~V} \\
B, 0.0 \mathrm{~V}\end{array}$ & $\begin{array}{l}X,-23.0 \mathrm{~dB}, \\
\mathrm{Y}, 0 \mathrm{~dB}\end{array}$ \\
\hline
\end{tabular}

FIG. 8. Structure of PLZT optical switch.
(8.0V) to only electrodes B, called the bar state (a) and the optical signal is output in $\mathrm{Y}$ port by applying the voltage $(10.5 \mathrm{~V})$ to only electrodes $\mathrm{A}$, called the cross state (c). In the low-power splitter mode, the optical signal is output in both $\mathrm{X}$ and $\mathrm{Y}$ ports without applying any voltage $(0.0 \mathrm{~V})$ to either electrode $\mathrm{A}$ or $\mathrm{B}$, where the switch acts as a splitter (b).

Therefore, the ODN creatively uses the above three modes, while considering various users' requirements, including the number of users, the transmission distance between L-OLT and L-ONUs, the permissible optical power to each user, and the required transmission method i.e. unicast or multicast.

Performance Evaluation for power consumption in the TDM-based active optical access network: In order to realize the low-power consumption network, we formulated the Integer Linear Programming (ILP) problem to maximize the number of PLZT optical switches used as the low-power splitter mode in the network, while considering the transmission distance and the permissible optical power to each user. The power consumption data of each component used in the simulation is shown below. One OLT is $12.5 \mathrm{~W}$ [21]. The $1 \times 2,1 \times 4,1 \times 8$, and $1 \times 16$ PLZT optical switches consume $2.5,2.5,2.6,2.8 \mathrm{~W}$, including the driver $(2.48 \mathrm{~W})$ $[15,16]$, which has already been developed. The optical amplifier using Erbium Doped Fiber Amplifier (EDFA) consumes $8.0 \mathrm{~W}$ [21], where the nominal gain is $17 \mathrm{~dB}$. The power consumption of each ONU is not included because it is a common element in each network. There are two comparison networks. The first network is the 10G-EPON [22], where the maximum number of ONUs per OLT is 32 and the maximum transmission distance between OLT and ONUs is $20 \mathrm{~km}$ and the optical amplifiers are needed when extending the network. Several extended-reach of amplified passive optical networks (PONs) have been proposed [23-25]. However, we use the 10G-EPON [22] as a basic PON architecture, which has been already standardized. The second network is the active optical access network with only switch modes of the PLZT optical switch, where the maximum number of ONUs per OLT is 128 and the maximum transmission distance between OLT and ONUs is $40 \mathrm{~km} \mathrm{[14].}$

Figure 9 shows that the TDM-based deterministic active optical access network with switch and low-power splitter modes reduces the power consumption by up to $52 \%$, compared to the $10 \mathrm{G}-\mathrm{EPON}$, and by up to $33 \%$, compared to the active

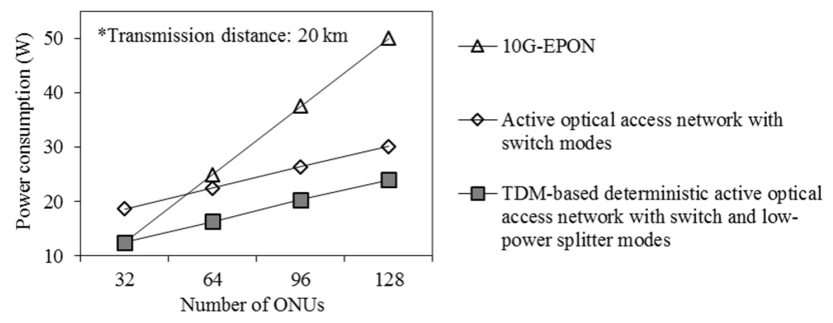

FIG. 9. Power consumption versus number ONUs. 


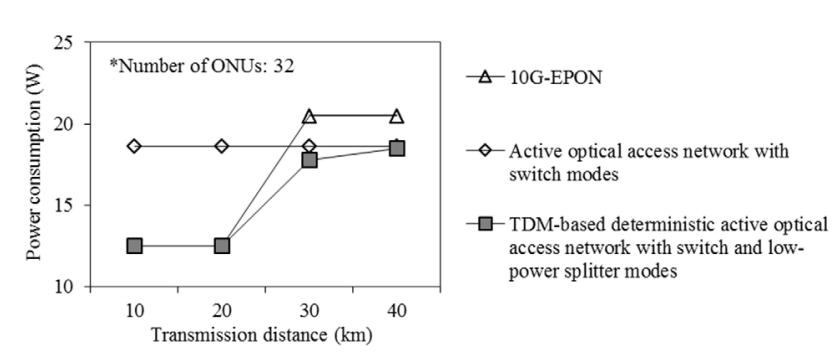

FIG. 10. Power consumption versus transmission distance.

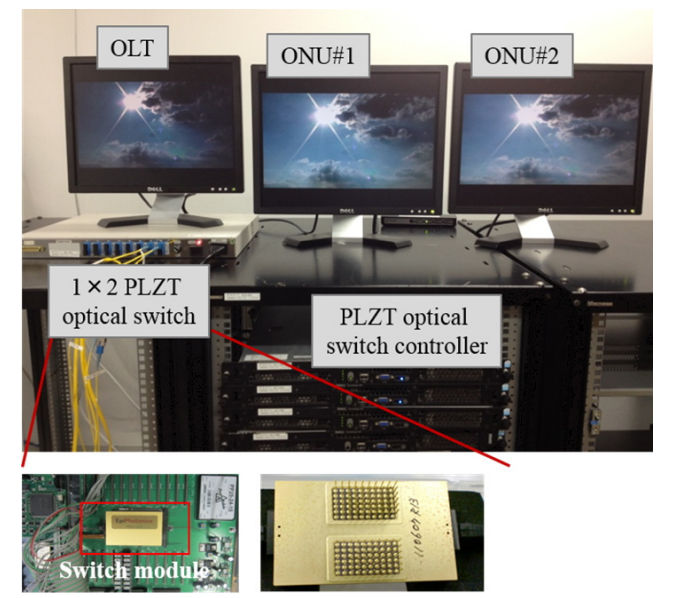

FIG. 11. A prototype of the TDM-based active optical access network.

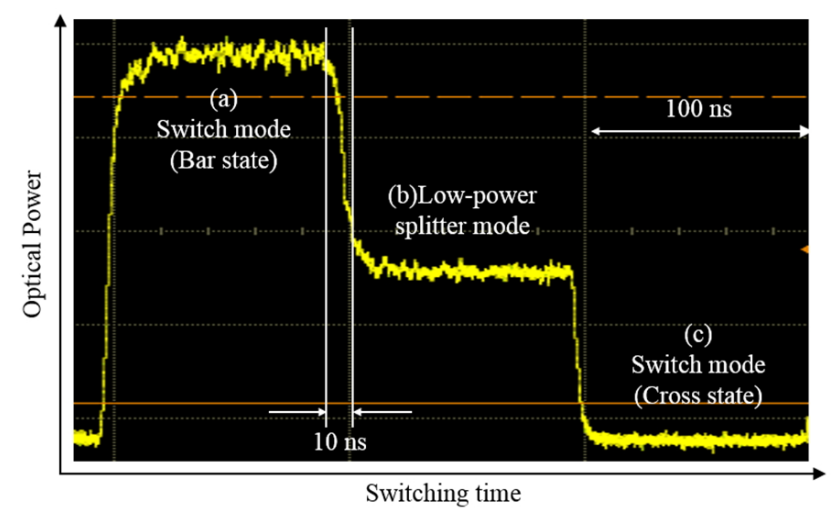

FIG. 12. Optical power versus switching time in each mode of the PLZT optical switch.

optical access network with only switch modes, where the transmission distance between OLT and ONUs is $20 \mathrm{~km}$. Figure 10 shows that the TDM-based deterministic active optical access network with switch and low-power splitter modes reduces the power consumption by up to $13 \%$, compared to the $10 \mathrm{G}-\mathrm{EPON}$, and by up to $33 \%$, compared to the active optical access network with only switch modes, where the number of ONUs is 32 .

In this way, the TDM-based active optical access network realizes an energy efficient architecture, compared to the
10G-EPON [22] and the active optical access network with only switch modes [14].

Experimental setup of the prototype of TDM-based deterministic active optical access network: The prototype of the active optical access network has been demonstrated in the developed experimental system in Fig. 11. There are one OLT, two ONUs, and one PLZT optical switch with switch and low-power splitter modes. Tests on the experimental system for supporting unicast and multicast by switching the switch mode and the low-power splitter mode at $10 \mathrm{~ns}$ confirmed the capability of the TDM-based deterministic active optical access network, as shown in Figs. 11 and 12.

\section{CONCLUSIONS}

This paper introduced a virtual optical slice core network and a TDM-based deterministic active optical access network for realizing ACTION. The virtual optical slice core can support multi QoS by using elastic optical pipes and provide an energy efficient network by adopting the MiDORi technique. Numerical results show that this core network adopting the MiDORi technique reduces the power consumption by up to $38 \%$, compared to without any power saving techniques. Also, the edge nodes of the core network, called ACTION edges, monitor application Quality of Experience (QoE) and assign flows to various multi-QoS slices. The TDM-based deterministic active optical access network can guarantee the various QoE requirements of each user in the access network based on QoS levels in the core network. It is possible to provide multi services and energy efficient network services by creatively using the switch and low-power splitter modes in the MZ type PLZT optical switch in the ODN. Numerical results show that this access network with switch and low-power splitter modes reduces the power consumption by up to $52 \%$, compared to the $10 \mathrm{G}-\mathrm{EPON}$ and by up to $33 \%$, compared to the active optical access network with only switch modes. Also tests on the experimental system for supporting unicast and multicast by switching the switch mode and the low-power splitter mode at 10ns confirmed the capability of the TDM-based deterministic active optical access network.

\section{ACKNOWLEDGMENT}

This work is supported by "ACTION Project" funded by the National Institute of Information and Communications Technology (NICT), Japan, and US NSF grants CNS-1405171 and CNS-1405405.

\section{REFERENCES}

1. Cisco Systems, Inc., "Cisco visual networking index: Forecast and methodology, 2013-2018," White Paper, June 2014. 
2. S. Jain, A. Kumar, S. Mandal, J. Ong, L. Poutievski, A. Singh, S. Venkata, J. Wanderer, J. Zhou, M. Zhu, J. Zolla, U. Hölzle, S. Stuart, and A. Vahdat, "B4: Experience with a globally-deployed software defined wan," in Proc. The ACM SIGCOMM 2013 Conference on SIGCOMM (SIGCOMM '13) (Hong Kong, China, Aug. 2013).

3. M. Pickavet, W. Vereecken, S. Demeyer, P. Audenaert, B. Vermeulen, C. Develder, D. Colle, B. Dhoedt, and P. Demeester, "Worldwide energy needs for ICT: the rise of power-aware networking," in Proc. IEEE Advanced Networks and Telecommunication Systems (ANTS) 2008 (Brussels, Belgium, Dec. 2008), pp. 1-3.

4. N. Yamanaka, S. Okamoto, E. Oki, A. Fumagalli, and M. Veeraraghavan, "Application-centric, energy-efficient network architecture, ACTION, based on flexible optical network," in Proc. COIN 2014 (Hyatt Regency Jeju, Jeju, Korea, Aug. 2014), No. TB1-3.

5. M. Jinno, H. Takara, B. Kozicki, Y. Tsukishima, Y. Sone, and S. Matsuoka, "Spectrum-efficient and scalable elastic optical path network: architecture, benefit, and enabling technologies," IEEE Comm. Mag. 47, 66-73 (2009).

6. O. Gerstel, M. Jinno, A. Lord, and S. J. B. Yoo, "Elastic optical networking: a new dawn for the optical layer?," IEEE Comm. Mag. 50, s12-s20 (2012).

7. G. Zhang, M. De Leenheer, A. Morea, and B. Mukherjee, "A survey on OFDM-based elastic core optical networking," IEEE Comm. Survey \& Tutorials 15, No. 1, First Quarter (2013).

8. N. Yamanaka, H. Takeshita, S. Okamoto, and S. Gao, "MiDORi: Energy efficient network based on optimizing network design tool, remote protocol and new layer-2 switch," in Proc. COIN 2010 (The Shilla Jeju, Jeju, Korea, July 2010), WeB1-1, pp. 353-355.

9. H. Yonezu, S. Gao, S. Shimizu, D. Ishii, S. Okamoto, E. Oki, and N. Yamanaka, "Network power saving topology calculation method by powering off links considering QoS," in Proc. OECC 2010 (Sapporo Convention Center, Sapporo, Japan, July 2010), No. 8P-12, pp. 586-587.

10. H. Yonezu, K. Kikuta, D. Ishii, S. Okamoto, E. Oki, and N. Yamanaka, "QoS aware energy optimal network topology design and dynamic link power management," in Proc. ECOC 2010 (Centro Congressi Lingotto, Torino, Italy, Sept. 2010), Tu.3.D.4.

11. H. Takeshita, Y. Oikawa, H. Yonezu, D. Ishii, S. Okamoto, and N. Yamanaka, "Demonstration of the self organized dynamic link power management by "MiDORi" energy optimal network topology design engine," in Proc. The Optical Fiber Communication Conference and Exposition (OFC) and the National Fiber Optic Engineers Conference (NFOEC) 2011 (Los Angeles Convention Center, Los Angeles, USA, March 2011), No. JWA060.

12. S. Okamoto, Y. Nomura, H. Yonezu, H. Takeshita, and N. Yamanaka, "GMPLS-enabled, energy-efficient, self-organized network: MiDORi," in Proc. Asia Comminications and Photonics Conference (ACP2011) (Shanghai International Convention Center, Shanghai, China, Nov. 2011), No. 8310-38.

13. A. Hirao, Y. Nomura, H. Takeshita, S. Okamoto, and N. Yamanaka, "Fast energy-efficient point-to-multipoint routing algorithm for MiDORi," in Proc. 10th Conference on Lasers and Electro-Optics Pacific Rim, and The 18th Opto Electronics and Communications Conference / Photonics in Switching 2013 (Kyoto International Conference Center, Kyoto, Japan, July 2013), No. MQ2-3.

14. K. Ashizawa, T. Sato, K. Tokuhashi, D. Ishii, S. Okamoto, N. Yamanaka, and E. Oki, "Scalable active optical access network using variable high-speed PLZT optical switch/ splitter," IEICE Transactions on Communications E95-B, 730-739 (2012).

15. K. Nashimoto, "PLZT waveguide devices for high speed switching and filtering," in Proc. OFC 2008 (San Diego Convention Center, San Diego, USA, 2008), OThE4.

16. EpiPhotonics Corp., http://epiphotonics.com/.

17. H. Ikeda, H. Takeshita, and S. Okamoto, "Future service adaptive access/aggregation network architecture," IEICE Transactions on Communications E95-B, 696-705 (2012).

18. H. Miyagi, Y. Okazaki, R. Usui, Y. Arakawa, S. Okamoto, and N. Yamanaka, "Performance evaluation of grid computing with parallel routes transmission," IEICE Transactions on Communications E91-B, 3882-3885 (2008).

19. Z. Yan, M. Veeraraghavan, C. Tracy, and C. Guok, "On how to provision virtual circuits for network-redirected largesized, high-rate flows," International Journal on Advances in Internet Technology 6, no 3 \& 4 (2013).

20. M. Veeraraghavan and I. Monga, "Broadening the scope of optical circuit networks," in Proc. IEEE ONDM 2014 (KTH Royal Institute of Technology, Kista Campus, Stockholm, Sweden, May 2014).

21. N. Yamanaka, H. Takeshita, K. Tokuhashi, and T. Sato, "Next generation green metro/access network architecture using time-slot-based optical aggregation network for multi-service access," Cyber Journals: Multidisciplinary Journals in Science and Technology 3, 7-12 (2013).

22. IEEE802.3-2012, "IEEE standard for ethernet," Clause 75-77, Dec. 2012, https://standards.ieee.org/findstds/standard/802.3-2012.html.

23. B. W. Kang and C. H. Kim, "Performance evaluation of bidirectional optical amplifiers for amplified passive optical network based on broadband light source seeded optical sources," J. Opt. Soc. Korea 15, 4-8 (2011).

24. B. W. Kang and C. H. Kim, "An amplified WDM-PON using broadband light source seeded optical sources and a novel bidirectional reach extender," J. Opt. Soc. Korea 15, 222-226 (2011).

25. B. W. Kang, K. Lee, S. B. Lee, and C. H. Kim, "Colorless amplified WDM-PON employing broadband light source seeded optical sources and channel-by-channel dispersion compensators for $>100 \mathrm{~km}$ reach," J. Opt. Soc. Korea 18, 436-441 (2014). 